\title{
CONTROLLED TOPOLOGY IN GEOMETRY
}

\author{
K. GROVE, P. PETERSEN V AND J. Y. WU
}

The purpose of the present note is to announce some finiteness theorems for classes of Riemannian manifolds (cf. $A, B$ and $D$ below).

Let $\mathscr{M}_{k, d, v}^{K, V}(n)$ denote the class of closed Riemannian $n$-manifolds with sectional curvatures between $k$ and $K$, diameter between $d$ and $D$, and volume between $v$ and $V$. Here $k \leq K$ are arbitrary, $0 \leq d \leq D$, and $0 \leq v \leq V$.

THEOREM A. For $n \neq 3,4$ the class $\mathscr{M}_{k, 0, v}^{\infty, D, \infty}(n)$ contains at most finitely many diffeomorphism types. sions.

This unifies and generalizes the following two theorems in high dimen-

Theorem (J. Cheeger [C, P]). The Class $\mathscr{M}_{k, 0, v}^{K, D, \infty}(n)$ contains at most finitely many diffeomorphism types.

Theorem (K. Grove, P. Petersen [GP]). The class $\mathscr{M}_{k, 0, v}^{\infty, D, \infty}$ contains at most finitely many homotopy types.

For $k>0$ and $n=3$, the conclusion in Theorem A follows by Hamilton's theorem in [H]. For $k>0$ and $n=4$ the fundamental group is either trivial or $\mathbf{Z}_{2}$ by Synge's theorem. Using Freedman's classification of simply connected topological 4-manifolds together with the above theorem and standard surgery theory then yields (cf. also [HK]).

CoROllary B. For $k>0$ the class $\mathscr{M}_{k, 0, v}^{\infty, \infty}(n)$ contains at most finitely many diffeomorphism (resp. homeomorphism) types when $n \neq 4$ (resp. $n=$ 4).

The basic construction in [GP] exhibits for each $M \in \mathscr{M}_{k, 0, v}^{\infty, D, \infty}(n)$ a suitable strong deformation retraction of an $a$ priori neighborhood of the diagonal in $M \times M$ onto the diagonal. This enables one to find $R, C>0$ so that for all $p \in M$ the metric $r$-ball $B(p, r)$ is contractible inside $B(p, C \cdot r)$ whenever $r \leq R$. This latter property carries over to any compact space $X=\lim M_{k}$ in the Gromov-Hausdorff closure of $\mathscr{M}_{k, 0, v}^{\infty, D, \infty}(n)$, moreover $\operatorname{dim} X \leq n$, cf. [PV]. Using the local contractibility properties, rather than the deformations as in [GP], one gets homotopy equivalences

$$
M_{k}^{\stackrel{f_{k}}{\longrightarrow}} X
$$

Received by the editors December 21, 1988.

1980 Mathematics Subject Classification (1985 Revision). Primary 53C20, 57N99.

The first two authors were supported in part by the National Science Foundation.

(C)1989 American Mathematical Society $0273-0979 / 89 \$ 1.00+\$ .25$ per page 
for large $k$, where diam $f_{k}=\sup \left\{\operatorname{diam} f_{k}^{-1}(x) \mid x \in X\right\} \rightarrow 0$ and diam $g_{k} \rightarrow$ 0 as $k \rightarrow \infty$, cf. [PV]. In particular, $X$ is $n$-dimensional.

It is a fairly easy consequence of results due to Begle [B] that $X=\lim M_{k}$ must be a homology manifold.

Combining all these properties of $X$ allows us to apply a result of F. Quinn [Q] to conclude that $X$ admits a resolution for $n \geq 4$. If in addition $n \geq 5$, and $X$ satisfies the disjoint disc property (DDP), it must be a topological manifold according to a theorem of R. D. Edwards, cf. [E, D]. To see that $X=\lim M_{k}$ indeed satisfies the DDP, one uses the deformations associated with $M_{k}$ together with the homotopy equivalences $f_{k}, g_{k}$. Hence

TheOREM C. For $n \geq 5$ any compact metric space in the GromovHausdorff closure of $\mathscr{M}_{k, 0, v}^{\infty, D, \infty}(n)$ is a topological n-manifold.

Having shown that $X$ is a topological manifold of dimension $\geq 5$ a result of T. A. Chapman and S. Ferry $[\mathbf{C F}, \mathbf{F}]$ implies that for $k$ sufficiently large $g_{k}$ can be deformed to a homeomorphism. Since the closure of $\mathscr{M}_{k, 0, D, \infty}^{\infty, D}(n)$ is compact, cf. [G], we conclude that this class contains at most finitely many homeomorphism types. By a general result of R. Kirby and $\mathrm{L}$. Siebenmann [KS], Theorem A follows.

The same argument as outlined above yields finiteness for diffeomorphism types rather than homotopy types in a finiteness theorem by T. Yamaguchi, cf. [Y]. In particular,

THEOREM D. For $n \geq 5$ the class of closed $n$-manifolds with injectivity radius bounded from below and volume from above, contains at most finitely many diffeomorphism types.

ACKNOWLEDGements. We would like to thank several people for their interest and suggestions concerning this work, in particular K. Corlette, S. Ferry, I. Hambleton, M. Rothenberg, D. Ruberman, and F. Quinn.

\section{REFERENCES}

[B] E. G. Begle, Regular convergence, Duke Math. J. 11 (1944), 441-450.

[CF] T. A. Chapman and S. Ferry, Approximating homotopy equivalences by homeomorphisms, Amer. J. Math. 101 (1979), 583-607.

[C] J. Cheeger, Finiteness theorems for Riemannian manifolds, Amer. J. Math. 92 (1970), 61-74.

[D] R. J. Daverman, Decompositions of manifolds, Academic Press, New York, 1986.

[E] R. D. Edwards, The topology of manifolds and cell-like maps, Proc. Internat. Congr. Math. Helsinki 1978 (O. Lehto, ed.), Acad. Sci. Fenn., Helsinki, 1980, pp. 111-127. 582.

[F] S. Ferry, Homotoping $\epsilon$-maps to homeomorphisms, Amer. J. Math. 101 (1979), 567-

[G] M. Gromov, Groups of polynomial growth and expanding maps, Inst. Hautes Études Sci. Publ. Math. 53 (1981), 183-215.

[GP] K. Grove and P. Petersen V, Bounding homotopy types by geometry, Ann. of Math. (2) 128 (1988), 195-206.

[H] R. S. Hamilton, Three-manifolds with positive Ricci curvature, J. Differential Geom. 17 (1982), 255-306.

[HK] I. Hambleton and M. Kreck, On the classification of topological 4-manifolds with finite fundamental group, Math. Ann. 280 (1988), 85-104. 
[KS] R. Kirby and L. Siebenmann, Foundational essays on topological manifolds, smoothings and triangulations, Ann. of Math. Studies 88, Princeton Univ. Press, Princeton, N. J., 1977.

[P] S. Peters, Cheeger's finiteness theorem for diffeomorphism classes of Riemannian manifolds, J. Reine Angew. Math. 394 (1984), 77-82.

[PV] P. Petersen V, A finiteness theorem for metric spaces, J. Differential Geom. (to appear).

[Q] F. Quinn, An obstruction to the resolution of homology manifolds, Michigan Math. J. 34 (1987), 285-291.

[Y] T. Yamaguchi, Homotopy finiteness theorems for certain precompact families of Riemannian manifolds, Proc. Amer. Math. Soc. 102 (1988), 660-666.

Department of Mathematics, University of Maryland, College Park, MaryLAND 20742

Department of Mathematics, Princeton University, Princeton, New Jersey 08544

Department of Mathematics, University of Chicago, Chicago, Illinois 60637 
